\title{
MODELING OF A MICROMAGNETIC IMPRINTING PROCESS
}

\author{
O. D. Oniku, A. Garraud, E. E. Shorman, W. C. Patterson, and D. P. Arnold
}

Interdisciplinary Microsystems Group, University of Florida, Gainesville, FL, USA

\begin{abstract}
In this article, we present the magnetic modeling of a batchfabrication process to imprint microscale pole patterns in hardmagnetic thick films. The imprinting process enables the creation of complex magnetic field patterns from permanent magnetic layers. The selective magnetic imprinting and the resultant stray fields are modeled in a two-step process using COMSOL Multiphysics in conjunction with measured magnetic hysteresis behavior. The models are used to evaluate the process design space and to predict the resulting stray magnetic field distribution in a patterned, $10-\mu \mathrm{m}$-thick Co-Pt magnetic film.
\end{abstract}

\section{INTRODUCTION}

In the past decade, there has been substantial advancement in the synthesis of high-performance, thick-film magnetic materials intended for MEMS applications [1,2]. This advancement has spawned growing interest in the integration of microfabricated magnetic materials into functional microsystems devices. However, the ongoing evolution of magnetic MEMS demands development of processes to microfabricate more complex, multipole magnetic structures to create spatially varying microscale magnetic field-patterns for microactuators, vibrational energy harvesters, lab-on-a-chip, and other applications [2].

Permanent magnetic materials require poling to induce a magnetization in a specific direction. This poling process is typically achieved by applying a large, external pulsed magnetic field. For wafer-scale fabrication, this standard approach restricts the magnetization of all magnetic materials on a wafer to only one single direction. Various approaches to realize fine-pitch $(<1 \mathrm{~mm}$ wide) multi-pole magnetic patterns have been investigated. These methods include: using patterned electrical conductors [3] or soft magnetizing heads $[4,5]$ to create shaped magnetic fields or, using materials with differing coercivities [6] or thermally reduced coercivities [7]. Micro-assembly has also been investigated [8].

Differing from these previous approaches ([3-8]), a key advantage of the imprinting process developed in this work is the ability to create diverse, complex pole geometries at the wafer scale with microscale pole widths. The process uses a microfabricated [9] or a laser-machined [10] soft-magnetic material as a "magnetic mask" (or a flux concentrator) to locally amplify the amplitude of an externally applied, pulsed magnetizing field. This shaped field is used to selectively reverse prescribed regions of a pre-magnetized hard magnetic layer.

We have utilized this process to imprint sub-100 $\mu \mathrm{m}$ features in 10- $\mu \mathrm{m}$-thick Co-rich CoPt magnetic layer [10]. However, the experimentally measured stray field patterns (12 mT peak-peak) did not match the $\sim 40 \mathrm{mT}$ peak-peak flux density or shape of the expected north/south pole pattern. In this article, we present magnetic modeling of the imprinting process (1) to help improve our understanding of the process; (2) to create a tool for optimization of the process; and (3) for predicting the final stray fields of imprinted magnetic layers.

\section{PROCESS DESCRIPTION}

A schematic representation describing the imprinting process is shown in Fig. 1. First a magnetically hard magnetic layer (film) is fabricated on a substrate $\left(10-\mu \mathrm{m}\right.$-thick $\mathrm{Co}_{80} \mathrm{Pt}_{20}$ [11]) and premagnetized "up" using a conventional pulsed magnetic field from a magnetizing fixture. A magnetically soft magnetic mask is then placed in contact with the pre-magnetized magnetic layer, and a second pulsed magnetic field (reversal field) is applied in the "down" direction. The magnetic masks used here are $125-\mu$ m-thick Fe foils that were laser-machined with slits to create lines and spaces for creating stripe-like magnetization patterns.

Owing to the field concentrating effect of the soft magnetic mask, different field intensities are "felt" by various regions in the magnetic layer. Figure 2 shows a COMSOL simulation that illustrates the field concentrating effect of a soft magnetic mask (in this case, Fe). The field distribution along the mid-plane of the 10$\mu$ m-thick Co-Pt layer is plotted in Fig. 2 b. For a $0.5 \mathrm{~T}$ applied reversal field, a $\sim 0.65 \mathrm{~T}$ field amplitude is obtained in the $\mathrm{Fe}$ region, whereas a $0.35 \mathrm{~T}$ field amplitude is obtained in the air region.

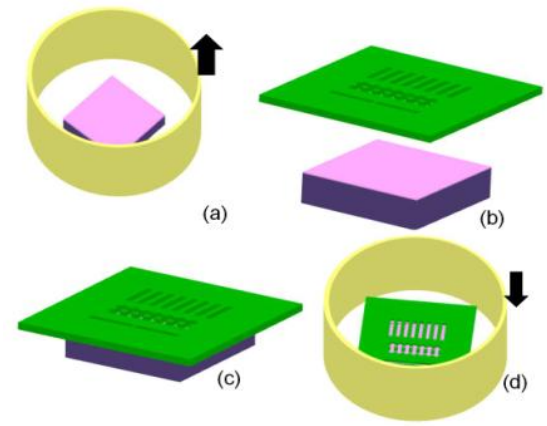

Figure 1: Magnetic patterning process: (a) initially magnetize a hard magnetic layer, (b) prepare magnetic mask by micromachining desired patterns on a soft magnetic foil, (c) bring mask in contact with magnetic layer, (d) apply a pulse reverse field.

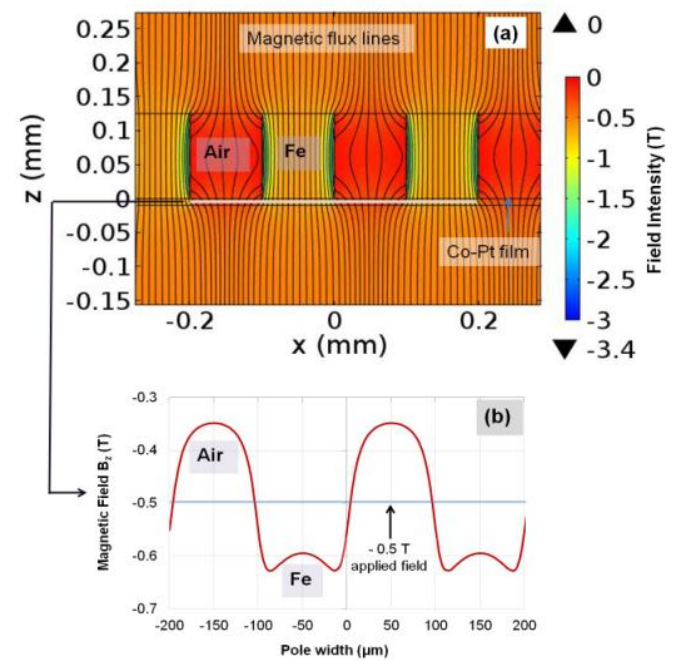

Figure 2: COMSOL simulation of the flux-concentrating effect of an Fe magnetic mask for a $0.5 \mathrm{~T}$ amplitude field applied in the downward direction: (a) cross-section distribution of magnetic flux density, (b) magnetic flux density along the mid-plane of the Co-Pt magnetic layer.

\section{MAGNETIC PROPERTIES}

For later use in finite-element models, the magnetic properties of both the soft Fe magnetic mask and the hard Co-Pt magnetic layer are measured using a vibrating sample magnetometer (VSM) 
(ADE Model EV9). Figure 3 presents the magnetic properties of the magnetic mask $\left(\mu_{0} M_{\text {sat }}=2.1 \mathrm{~T} ; \mu_{r}=800\right)$, with the inset showing its optical image. Figure 4 presents the full hysteresis loop along with recoil curves for the $10-\mu \mathrm{m}$-thick Co-Pt magnetic layer $\left(\mu_{0} H_{c i}=0.41 \mathrm{~T}\right)$ used in this work. The recoil curves are measured at various fields, corresponding to the applied reversal field amplitudes and will be useful for later modeling of the patterned magnetic structure. The inset is a plot showing the recoil remanence for various reverse fields.

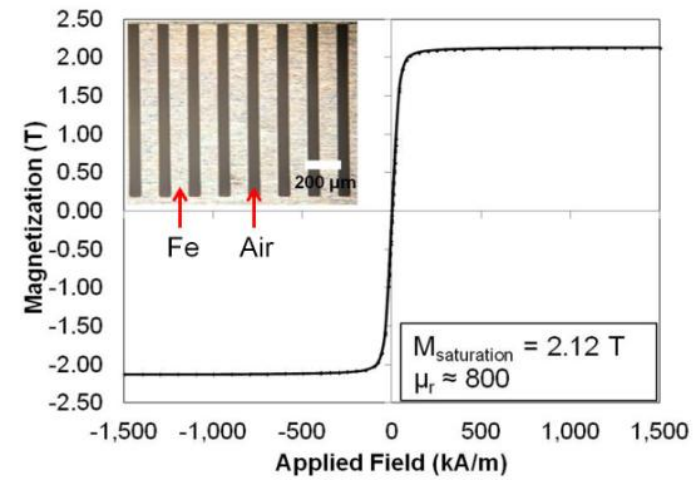

Figure 3: Magnetic properties of laser-micromachined softmagnetic Fe foil mask. Inset shows an optical image of the mask with a feature size of $100 \mu \mathrm{m}$.

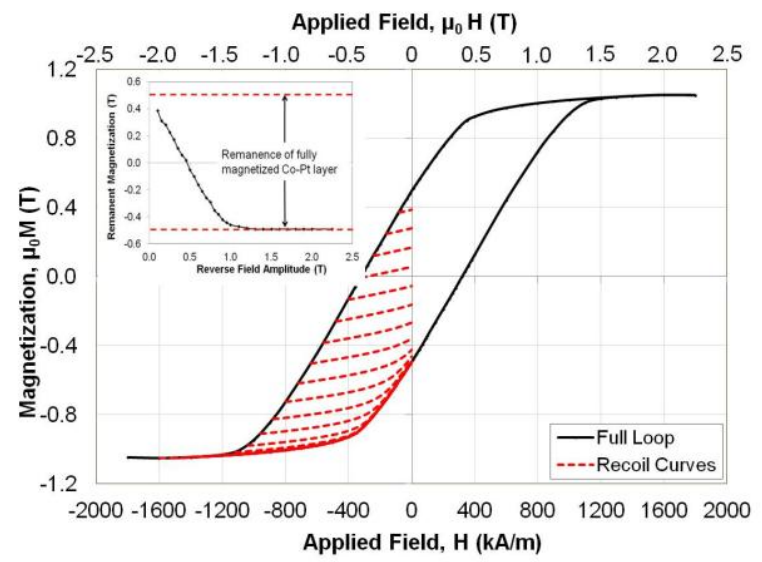

Figure 4: Magnetic properties of electroplated Co-Pt film showing recoil curves at various reversal fields. The inset shows the remanent magnetization of the Co-Pt layer as a function of the reversal field amplitude.

\section{MODELING}

The imprinting process is modeled in two stages using 2D magnetostatic finite-element simulations in COMSOL Multiphysics. The first stage simulates the shaped magnetic fields during the peak of the pulsed reversal field. The second stage simulates the final patterned magnetic layer after the imprinting process, making use of information from the first stage simulation. All simulations model the nonlinear magnetic properties using the measured magnetic hysteresis curves presented in Figs. 3 and 4.

\section{Stage 1: Simulation of Pulse Reversal Field}

First, the field-concentrating effects of the 125- $\mu \mathrm{m}$-thick Fe magnetic mask and a $10-\mu$ m-thick Co-Pt magnetic layer are simulated for various reversal field amplitudes. Figure 5 shows how the field amplitudes at the mid-plane of the Co-Pt layer (i.e. 5 $\mu \mathrm{m}$ below the mask) evolve with the reversal field. The data points and connecting lines represent the field at the mid-point (center) underneath the Fe and air, respectively. The error bars represent the peak fields of the "bunny ear" features that arise due to edgeeffects under the Fe regions (refer to Fig. 2b).

For good pattern transfer, the intrinsic coercivity $\left(H_{c i}\right)$ of the hard magnetic layer must lie in between the field amplitudes in the air and that in the Fe regions, i.e. $\left|B_{\text {air }}\right|<\left|H_{c i}\right|<\left|B_{F e}\right|$. This ensures that the field in the Fe region is sufficient to flip the magnetization, whereas the field in the air region is not strong enough. Another consideration is the field-contrast

$$
\gamma=\frac{B_{F e}-B_{a i r}}{B_{F e}+B_{a i r}}
$$

which quantifies the difference between the B-field intensities in the $\mathrm{Fe}$ and air regions, and should be maximized for good pattern transfer. As shown in Fig. 6, the field contrast appears to be fairly constant for all applied reverse field amplitudes until reaching the saturation level of the magnetic mask (2.1 $\mathrm{T}$ in this case).

These simulations suggest a reversal field amplitude of $\sim 0.5$ $\mathrm{T}$, which correlates well with the reversal field amplitude found previously to yield good pattern transfer [10]. Example magnetic patterns are shown in Fig. 7.

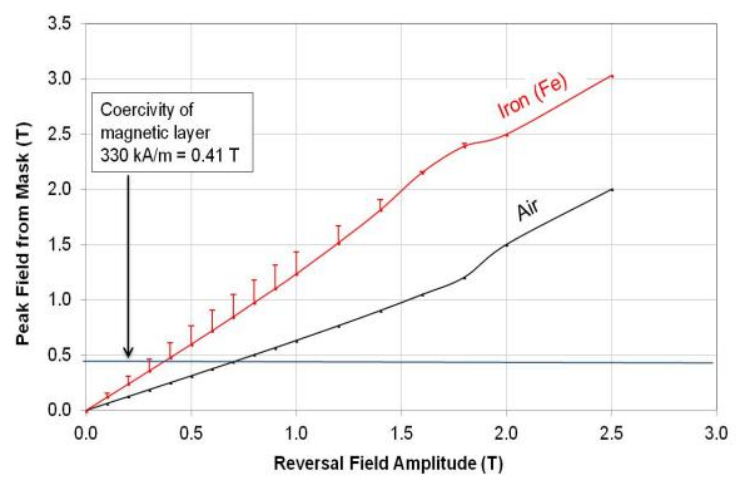

Figure 5: COMSOL simulation of field amplitudes in the $\mathrm{Fe}$ and

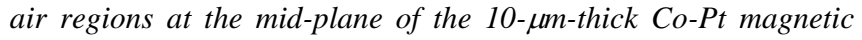

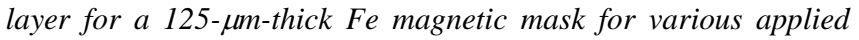
pulse reverse fields.

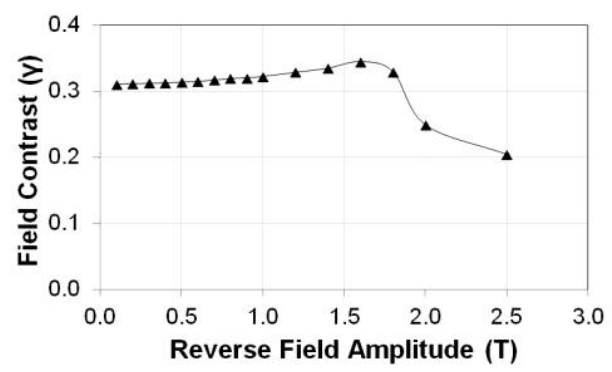

Figure 6: Field contrast for the data shown in Figure 5 as a function of applied reverse field.

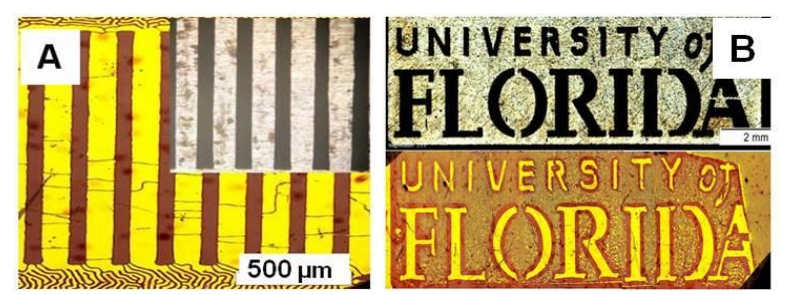

Figure 7: Optical images of magnetic masks and resulting patterned magnetic layers (magneto-optical images) showing good pattern transfer using a 0.5-T reversal field: (a) simple stripe-like

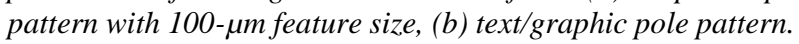



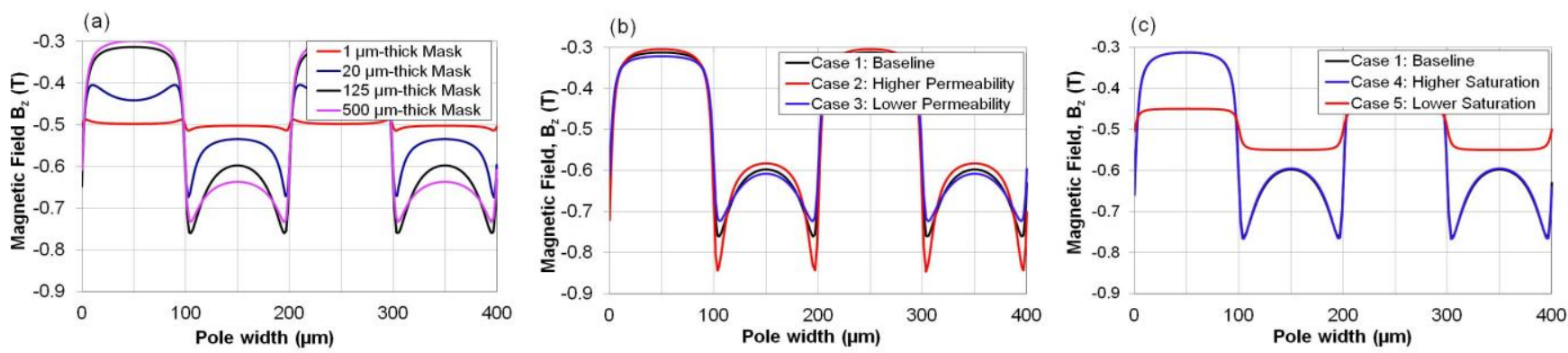

Figure 8: Study of the effects of mask properties and geometry for a 0.5 T reversal field: (a) effect of mask thickness. (b) effect of saturation magnetization, and (c) effect of relative permeability. All simulations are for a 10- $\mu$ m thick Co-Pt magnetic layer.

To further study the field concentrating effects of the magnetic mask, additional simulations are performed with parametric variations of the mask geometry and mask magnetic properties. Table 1 summarizes the different magnetic properties considered in this study. Figure 8 shows different parametric variations of the magnetic mask, assuming a $0.5 \mathrm{~T}$ pulse reversal field and a 10- $\mu$ m-thick Co-Pt magnetic layer.

Table 1: Magnetic properties of the magnetic mask considered in the parametric simulations.

\begin{tabular}{|l|c|c|c|c|c|}
\hline \multicolumn{1}{|c|}{ Case } & $\begin{array}{c}\mathbf{1} \\
\text { (Baseline) }\end{array}$ & $\mathbf{2}$ & $\mathbf{3}$ & $\mathbf{4}$ & $\mathbf{5}$ \\
\hline $\mathrm{M}_{\mathrm{sat}}(\mathrm{T})$ & 2.1 & 2.1 & 2.1 & 3.0 & 0.2 \\
\hline$\mu_{\mathrm{r}}$ & 800 & 10000 & 50 & 800 & 800 \\
\hline
\end{tabular}

Figure $8 \mathrm{a}$ indicates that in order to obtain a good pattern transfer, the thickness of the magnetic mask should be significantly greater than the thickness of the hard magnetic film. If the mask is too thin (e.g. the $1 \mu \mathrm{m}$ case), there is little field contrast in the underlying magnetic layer. In the case considered here, the mask thickness $(125 \mu \mathrm{m})$ is $12 \mathrm{x}$ greater than the thickness of the magnetic film $(10 \mu \mathrm{m})$, which yields about $80 \%$ of the maximum field contrast.

Figure $8 \mathrm{~b}$ shows that while the relative permeability of the magnetic mask does not significantly affect the field contrast, a higher permeability will produce more pronounced edge effects that increase the non-uniform field distribution ("bunny ears") in the $\mathrm{Fe}$ region. (In this case the mask is not saturated)

Figure 8c shows that the saturation magnetization of the magnetic mask appears to be a significant factor in the field contrast - a high saturation material yields a larger field contrast and vice versa. Figure 9 studies this effect with more detail, showing the predicted shaped fields for various mask saturation values. It is observed that when the saturation magnetization of the mask is at below the pulse reversal field $(0.5 \mathrm{~T})$-meaning the mask is saturated - the field distribution in the $\mathrm{Fe}$ and air regions is fairly smooth and symmetric, with a peak-peak variation approximately equal to half the saturation magnetization of the mask. When the mask has a higher saturation-and is not saturated - the field pattern becomes more non-uniform, i.e. the "bunny ears" get larger and larger.

\section{Stage 2: Simulation of Imprinted Magnetic Layer}

To further explore the modeling of the selective imprinting process, a second model is developed to predict the resultant stray fields from the selectively magnetized layer. For this model, the magnetic layer is sub-divided into discrete sectors that describe the spatial field distribution from the mask in a piecewise fashion.

Figure 10 shows an example of the piecewise representation. The magnetic properties of each sector are defined by a specific recoil-magnetization curve (shown in Fig. 4), which depends on

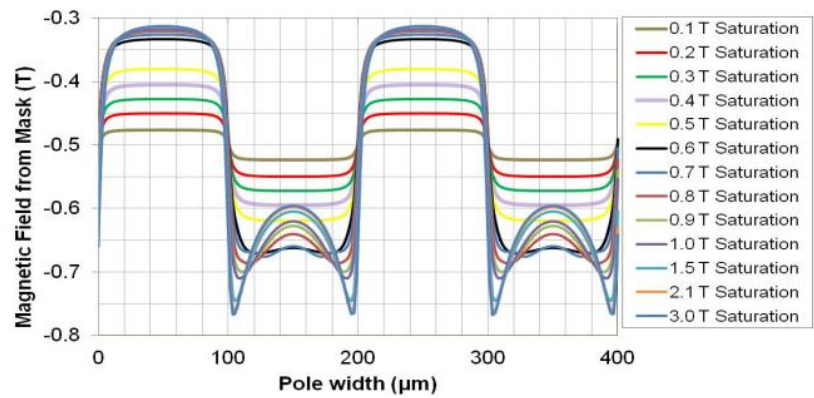

Figure 9: Magnetic field distribution from masks with various saturation magnetizations $\left(M_{\text {sat }}\right)$ using a 0.5-T reversal field. Result indicates the disappearance of the "bunny-ear" shape effect for materials with $M_{\text {sat }}$ close to or lower than the applied reversal field. (All results are evaluated at $5 \mu \mathrm{m}$ distance from the mask).

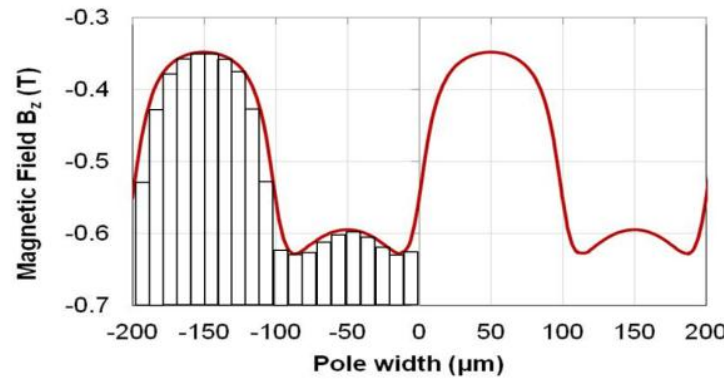

Figure 10: Piecewise representation of the mask field distribution where each discrete sector represents the amplitude and position of the field felt during the selective magnetization process. Ten sectors (each 10- $\mu m$ wide) are used to represent the field distribution in each of the Fe and air regions.

the amplitude and position of the field experienced during the reversal process. By simulating the ensemble of these sectors, the net magnetic field from the multi-pole patterned layer can then be evaluated at various heights.

Using this method, Fig. 11 shows the simulated stray magnetic flux densities at various heights above the surface for a $10-\mu$ m-thick Co-Pt film patterned using the $125-\mu \mathrm{m}$-thick $\mathrm{Fe}$ magnetic mask with a $0.5 \mathrm{~T}$ pulse reversal field. Nearer to the surface of the magnetic layer, there is evidence of the non-uniform magnetization in the $\mathrm{Fe}$ regions (the "bunny ears"). As the evaluation height is farther away from the surface of the patterned magnetic layer, the field distribution becomes more uniform and sinusoidal. As indicated earlier, this non-uniformity arises from the fact that the saturation magnetization of the Fe magnetic mask $(2.1 \mathrm{~T})$ is significantly higher than the applied reversal field $(0.5 \mathrm{~T})$. 


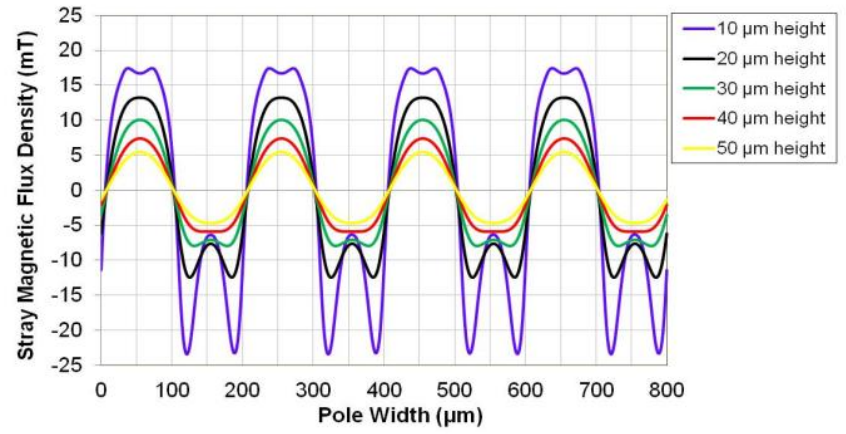

Figure 11: COMSOL simulation of stray magnetic flux density from patterned magnetic film using the modeling approach described in the article.

Furthermore, a comparison is made between the COMSOL simulations and experimental field measurements obtained from a quantitative magneto-optical indicator film (MOIF) [12]. Figure 12 shows the measured fields in comparison to the fields predicted by our process model and the expected fields for an "ideal" magnetization (where each pole is uniformly magnetized to its remanent value). The simulated data matches quite well in both amplitude and shape to the measured field pattern. In contrast, the fields predicted for the ideal magnetization significantly overpredict the amplitude and do not capture the shape of the real field pattern. The difference between the ideal results and the actual results are due to the imperfect magnetization, as described earlier. The complex shape of the measured and simulated stray field is attributed to the field shaping of the mask.

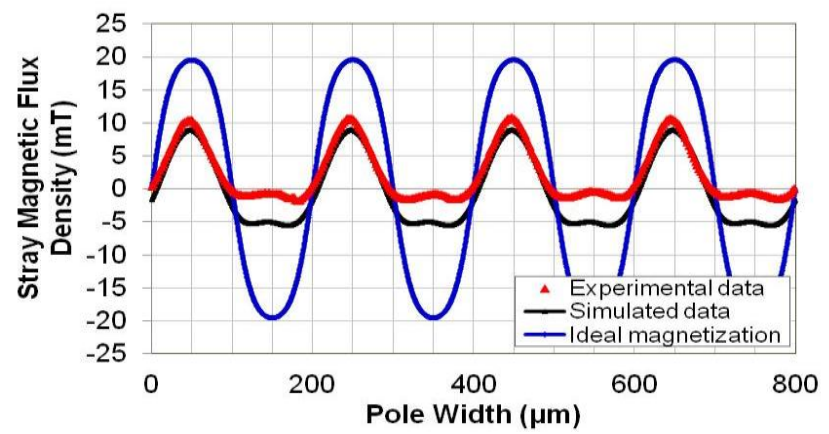

Figure 12: Comparison of measured stray magnetic flux density with simulated results at $\sim 35 \mu \mathrm{m}$ above the surface of a patterned Co-Pt magnetic layer.

\section{CONCLUSION}

We have introduced a modeling approach for a microscale magnetic imprinting process. This model provides a tool for the prediction of the stray magnetic field and the optimization of the magnetic pattern transfer. The pulsed field amplitude required for the process is determined by the magnetic properties of the magnetic layer to be patterned. However, simulations show that the thickness and saturation magnetization of the magnetic mask are important factors to be considered to obtain a good field concentrating contrast and consequently a desirable pattern transfer. We show that the magnetic mask thickness should be at least 10x greater than the thickness of the magnetic layer, and the saturation magnetization should be approximately equal to the required reversal field amplitude. The relative permeability of the magnetic mask is not so important, so long as it is significantly greater than that of air.
The modeling process is experimentally evaluated by patterning simple stripe patterns in 10 - $\mu$ m-thick Co-Pt film using a $125-\mu \mathrm{m}$-thick $\mathrm{Fe}$ foil magnetic mask. A stray magnetic flux density of $12 \mathrm{mT}$ peak-peak is measured above the patterned film, showing reasonable agreement in both amplitude and shape with the model simulations.

\section{ACKNOWLEDGEMENT}

This work was supported in part by US Army Research Office (Grant \#W911NF-09-1-0511) and DARPA (Grant \#N6601-11-14198).

\section{REFERENCES}

[1] D. P. Arnold, and N. Wang, "Permanent Magnets for MEMS," J. Microelectromechanical Systems, vol. 18, no. 6, pp. 1255-1266, 2009.

[2] N. M. Dempsey, "Hard Magnetic Materials for MEMS Applications," Nanoscale Magnetic Materials and Applications, J. P. Liu, E. Fullerton, O. Gutfleisch et al., eds., pp. 661-680: Springer, 2009.

[3] J. Töpfer, B. Pawlowski, H. Beer et al., "Multi-pole magnetization of $\mathrm{NdFeB}$ magnets for magnetic microactuators and its characterization with a magnetic field mapping device," J. Magnetism and Magnetic Mat., vol. 270, no. 1-2, pp. 124-129, 2004.

[4] I. Zana, F. Herrault, D. P. Arnold, and M. Allen, "Magnetic patterning of permanent-magnet rotors for microscale motor/generators," PowerMEMS 2005, Tokyo, Japan, 2005, pp. 116-119.

[5] O. D. Oniku, R. Regojo, Z. A. Kaufman, W. C. Patterson and D. P. Arnold, "Batch magnetic patterning of hard magnetic films using pulsed magnetic fields and soft magnetizing heads," IEEE Trans. Magnetics, vol. 49, no. 7, pp. 4116-4119, 2013.

[6] F. M. F. Rhen, E. Backen, and J. M. D. Coey, "Thick-film permanent magnets by membrane electrodeposition," J. Appl. Phys., vol. 97, no. 11, pp. 113908-4, 2005.

[7] F. Dumas-Bouchiat, L. F. Zanini, M. Kustov et al., "Thermomagnetically patterned micromagnets," Appl. Phys. Lett., vol. 96, no. 10, pp. 102511-3, 2010.

[8] B. A. Peterson, W. C. Patterson, F. Herrault, D. P. Arnold and M. G. Allen ., "Laser-micromachined Permanent Magnet Arrays with Spatially Alternating Magnetic Field Distribution, PowerMEMS 2012, Atlanta, GA, pp 319-322.

[9] A. Garraud, O. D. Oniku, W. C. Patterson, E. Shorman, D. LeRoy, N. Dempsey, and D. P. Arnold, "Microscale Magnetic Patterning of Hard Magnetic Films Using Microfabricated Magnetizing Masks," MEMS 2014, San Francisco, CA, 2014.

[10] O. D. Oniku, P. V. Ryiz, A. Garraud, and D. P. Arnold, "Imprinting of fine-scale magnetic patterns in electroplated hard magnetic films using magnetic foil masks," J. Appl. Phys., vol. 115, 2014.

[11] O. D. Oniku, and D. P. Arnold, "Microfabrication of HighPerformance Thick Co80Pt20 Permanent Magnets for Microsystems Applications," ECS Trans., vol. 50, no. 10, pp. 167-174, 2013.

[12] W. C, Patterson, E. E. Shorman, N. Garraud, and D. P. Arnold, "A Magnetic Microscope for Quantitative Mapping of the Stray Fields from Magnetic Microstructures" Hilton Head 2014, Parallel publication,

\section{CONTACT}

*O. D. Oniku, tel: +1-352-514-5820; ololadeoniku@ufl.edu 